\title{
Aplicación de tecnología en la práctica de la formación inicial de profesores. Valoración del análisis didáctico de clases de enseñanza secundaria filmadas en $360^{\circ 1}$
}

\author{
Application of technology in the practice of initial teachers training. \\ Assessment of didactic analysis for secondary education classrooms \\ filmed in $360^{\circ}$
}

\section{Aplicação de tecnologia na prática da formação inicial de professores. Avaliação da análise didática das aulas de ensino médio filmadas em $360^{\circ}$}

ISSN 1688-9304 - DOI: 10.18861/cied.2020.11.2.2993

Silvia Umpiérrez Oroño*1

https://orcid.org/0000-0003-3341-9687

Delma Cabrera**2

https://orcid.org/0000-0002-8506-4489

Paola Bruccoleri***3

https://orcid.org/0000-0003-1012-8000

Fecha de recibido: 21/01/2020

Fecha de aprobado: 24/06/2020

\section{Resumen}

La formación inicial de profesores de enseñanza secundaria en Uruguay prevé actividades de observación y análisis didáctico de clases en centros educativos de dicho nivel, de forma presencial y fundamentalmente individual. Con el objetivo de enriquecer y diversificar esta formación, la presente investigación propuso evidenciar las percepciones de docentes y estudiantes y estudiar los niveles de reflexión alcanzados por medio de la observación de clases filmadas en $360^{\circ}$ y de la incorporación de modalidades de trabajo asincrónicas, virtuales, interdisciplinares y grupales. La metodología se basó en un diseño cualitativo. Incluyó seis instancias cuasi-experimentales en las que estudiantes $(n=61)$, distribuidos en seis grupos, orientados por uno o varios formadores docentes de didáctica $(n=8)$ realizaron observación y análisis didáctico de unidades fílmicas en $360^{\circ}$. Se recolectaron datos por medio de observación no participante de las instancias cuasi-experimentales, análisis de contenido de foros, correos electrónicos y documentos con producciones de los estudiantes, así como de 39 encuestas y 8 entrevistas en profundidad a estudiantes y formadores. Los formadores y estudiantes participantes valoran favorablemente el recurso como un complemento a la modalidad de formación actual y opinan que propician el análisis didáctico y la reflexión. Las unidades fílmicas se adecuan a los objetivos de los formadores, a variadas modalidades de trabajo y a la diversidad de formas de aprender. Encuentran que se propició la flexibilidad horaria de la formación y la colaboración. Se alcanzaron variados niveles de reflexión pero no el de mayor profundidad. Es necesario completar el estudio para comprender las razones de ello. Se ha creado un sitio Web con un repositorio abierto con las unidades fílmicas (http://recyreap.cfe.edu.uy) y se ha 
dispuesto, junto a ellas, la variedad de protocolos diseñados por el equipo, que podrían servir para guiar el análisis didáctico y la reflexión.

Palabras clave: tecnología, formación inicial de profesores, innovación educativa, filmación.

\section{Abstract}

The initial training of secondary school teachers in Uruguay includes activities of observation and didactic analysis of classrooms in educational centers of this level, in face-to-face and mainly individual modalities. With the objective of enhancing and diversifying this training, the ongoing research proposed to make visible the perceptions of teachers and students, and to analyze the levels of reflection achieved, through the observation of lessons filmed in $360^{\circ}$, as well as the incorporation of class works that are asynchronous, virtual, interdisciplinary and in groups. The methodology was based on a qualitative design. It included six quasi-experimental instances where students $(n=61)$ were distributed in six groups, oriented by one or several teaching trainers $(n=8)$, and performed observation and didactic analysis of the film units in $360^{\circ}$. Data were collected through non-participant observation of quasi-experimental instances, content analysis of forums, e-mails and documents with student productions, as well as 39 surveys and 8 in-depth interviews to students and trainers. Both trainers and students that participated in this research favorably valued the resource as a complement to the current training modality, and expressed that it promotes didactic analysis and reflection. The film units fit the trainers' targets, the various work modalities and the diverse ways of learning. They found that both flexibility of training time and collaboration were fostered. Various levels of reflection were reached, although the deepest level was not attained. It is necessary to complete the study to understand the reasons for it. A website with an open repository with the film units (http://recyreap.cfe.edu.uy) was created, and a number of protocols designed by the team, which could serve as a guide for didactic analysis and reflection, are also offered.

Keywords: technology, initial teacher training, educational innovation, filming

\section{Resumo}

A formação inicial de professores de ensino médio no Uruguai prevê atividades de observação e análise didática de aulas em centros educacionais desse nível, em modalidade presencial e fundamentalmente individual. Com o objetivo de enriquecer e diversificar esse treinamento, a pesquisa propôs mostrar as percepções de professores e alunos, e estudar os níveis de reflexão alcançados, observando aulas filmadas em 360 graus, incorporando modalidades de trabalho assíncronas, virtuais, interdisciplinares e de grupo. A metodologia foi baseada em um desenho qualitativo. Foram incluídas seis instâncias quase-experimentais em que os alunos $(n=61)$, distribuídos em seis grupos, guiados por um ou mais instrutores didáticos $(n=8)$, realizavam observação didática e análise de unidades fílmicas de $360^{\circ}$. Os dados foram coletados através da observação não participante de instâncias quase-experimentais, análise de conteúdo de fóruns, e-mails e documentos com produções de alunos, 39 pesquisas e 8 entrevistas em profundidade com estudantes e treinadores. Os treinadores e estudantes participantes valorizam favoravelmente o recurso como um complemento à atual modalidade de treinamento e acreditam que promovem análise e reflexão didática. As unidades do filme mostraram se adaptar aos objetivos dos treinadores, às várias modalidades de trabalho e à diversidade de formas de aprendizado. Eles acham que a flexibilidade do treinamento e a colaboração foram incentivadas. Vários níveis de reflexão foram alcançados, mais não o mais profundo. É necessário completar o estudo para entender as razões disso. Um site 
Aplicación de tecnología en la práctica de la formación inicial de profesores. Valoración del análisis...

com um repositório aberto com as unidades fílmicas foi criado (http://recyreap.cfe.edu. uy) e uma variedade de protocolos foram gerados pela equipe, os que podem servir para orientar análise e reflexão didática.

Palavras-chave: tecnologia, formação inicial de professores, inovação educacional, filmagem.

\section{Introducción}

El análisis didáctico se desarrolla mediante la observación de una instancia de enseñanza durante la formación inicial de profesores. El componente reflexivo del análisis permite profundizar sobre las dimensiones de la práctica de enseñanza, incluidas la política y la ética. También habilita a capitalizar experiencias que han adquirido otras personas, propicia las miradas sobre uno mismo como aprendiz, contribuye a la comprensión sobre la complejidad de la actividad educativa y favorece la cooperación (Perrenoud, 2007).

La formación inicial de profesores de enseñanza secundaria (o media, para Uruguay) incluye actividades de observación y análisis didáctico de clases en centros educativos de dicho nivel de forma presencial, sincrónica y fundamentalmente individual. Cada estudiante cuenta con un profesor adscriptor, que es el titular del grupo de enseñanza media donde se realiza, durante todo el año, esta formación denominada Unidad Didáctica Práctica. Por una parte el estudiante fundamentalmente observa al docente adscriptor, tiene acceso a sus planificaciones y realiza, supervisado por este, algunas actividades de enseñanza. Por otra parte, el formador de didáctica es el docente que lleva adelante las clases de didáctica teórica en el instituto de formación docente correspondiente o por medio de plataforma digital educativa. También realiza visitas para observar al practicante en actividades de enseñanza media, entre tres y cinco veces al año. Luego de observar la clase dictada por el estudiante realiza una devolución, presencial o virtual, donde se vuelcan los comentarios y materiales que puedan ayudar en los aprendizajes didácticoprácticos del estudiante. Este formato no prevé ni propicia instancias de análisis didáctico en las que los estudiantes, desde varias disciplinas, en diferentes modalidades de participación e incluso en diferentes grados de avance de la carrera, puedan intercambiar reflexiones sobre prácticas de enseñanza de más de un docente.

Con el objetivo de enriquecer y diversificar esta formación, la presente investigación propuso valorar nuevas modalidades de trabajo, asincrónicas, virtuales, interdisciplinares y grupales, entre otras, utilizando clases filmadas en $360^{\circ}$ en centros de enseñanza media. La investigación busca recoger las percepciones de los usuarios (docentes y estudiantes) y evidenciar los niveles de reflexividad alcanzados en el análisis didáctico de dichas clases.

\section{Marco teórico}

Este proyecto se inscribe en el campo de la innovación pues intenta valorar dispositivos de formación docente en una modalidad disruptiva en relación a las existentes en el país pero que, a la vez, pueda articularse con estas. No busca sustituirlas sino hallar alternativas que enriquezcan las prácticas tradicionales. Para que esa articulación se produzca es necesario tener en cuenta qué aspectos de la innovación son seductores, con el fin de mejorar las posibilidades de su incorporación. Vaillant (2019) enumera algunas de las características de los dispositivos de innovación exitosos, tales como: que puedan ser utilizados tanto por docentes en formación como en servicio, que propendan al trabajo colaborativo, que vinculen la teoría con la práctica, que permitan la interacción de personas en diferentes niveles de la profesión -desde estudiantes hasta docentes expertos- y que incorporen el componente reflexivo.

La investigación persigue la idea de una formación no reproductiva, donde el foco 
se centre en "el desarrollo de capacidades para la práctica docente en situaciones y contextos reales" y complejos (Davini, 2015, 13). Las tradiciones normalista, académica y tecnicista sostienen la idea de que la práctica es un campo de aplicación de métodos y conocimientos. Dichas tradiciones tuvieron un alto impacto en el desarrollo de la didáctica y una fuerte influencia en las prácticas. Sin embargo, actualmente se adjudica mayor significado a la concepción del docente que, a partir de un compromiso con un determinado habitus profesional, toma decisiones continuamente y, para ello, reflexiona. El profesional reflexivo es quien desempeña "un rol activo en la formulación de sus objetivos y métodos de enseñanza, en contraposición al profesor que administra y ejecuta propuestas técnicas diseñadas desde el exterior de las aulas" (Anijovich y Cappelletti , 2018, 25).

Sáez y Cortés $(2019,130)$ plantean que "a pesar de la cantidad de estudios centrados en la reflexión son pocos los que intentan registrarla de algún modo". Caracterizan a la reflexión como un proceso en el que se crean significados que otorgan al estudiante mayor comprensión de las conexiones con otras experiencias similares, que implica el pensamiento riguroso, sistemático y anclado en la investigación científica, que se produce en comunidad y que requiere valorar tanto el crecimiento personal como el del colectivo con el que interactúa.

Tal como afirma Perrenoud (2007), el vínculo entre análisis y reflexión se presenta al pensar sobre la acción, a partir de los aspectos conscientes, sus restricciones y oportunidades. De tal forma los dispositivos de formación pueden ser muchos dado que existen varias líneas teóricas que convergen en la reflexión. No obstante, la misma no es un intento de regular racionalmente la acción profesional sino hacer que los estudiantes se contacten con el accionar profesional, con la confianza en la educabilidad de las personas como principal aliciente. No se trata solo de pensar en cómo se podría haber hecho algo mejor sino en comprender por qué se hizo así.

El uso de videos ha sido identificado por algunos autores como una herramienta que permite la toma de distancia del hecho analizado y se integra a dispositivos de prolongación de la actividad constructiva de los aprendices (Pereyra, Moscato, Calderón y Oviedo, 2016). Este distanciamiento permite "hurgar en las heridas sin provocar demasiados daños" (Perrenoud, 2007, 130) y analizar reflexivamente el trabajo de otro docente voluntario mientras la autoestima del estudiante se fortalece por medio de los aprendizajes y de su puesta en práctica. Es decir, dar tregua a la actividad más frecuente de la práctica preprofesional, tal como se practica en el país, que es analizar las propias actividades de enseñanza o las del mismo docente adscriptor durante todo el año. Esto se constituye en un nuevo formato de observación y análisis de situaciones reales que les sucedieron a otros, en otro momento y en otro lugar, que pueden recuperarse y traerse a clase para reflexionar. Se suscitan oportunidades casi de laboratorio, minimizando los riesgos y con la posibilidad de repetir el análisis en condiciones de seguridad y control (Fernández y Fernández, 1994; Fernández, Elórtegui y Medina, 2003; Anijovich y Cappelletti, 2018).

Según investigaciones actuales, la introducción de las tecnologías digitales en el aula de educación media aporta favorablemente a la alfabetización digital de los alumnos. La formación ciudadana, el acceso a procesos de ascenso social y el éxito académico están ligados a las competencias digitales de las personas. El desarrollo de dichas competencias debe propiciarse desde la educación primaria y media y, para esto, se requiere de docentes preparados. Sin embargo, si estos ven a las tecnologías digitales como una imposición porque no se han formado con y en las mismas, los resultados suelen ser magros en el desarrollo de las competencias digitales de los alumnos (Fernández, 2016). 
Aplicación de tecnología en la práctica de la formación inicial de profesores. Valoración del análisis...

La inclusión digital genuina implica:

a) que la incorporación de las tecnologías digitales sea natural, no forzada, al utilizarlas en el marco de instancias curriculares preexistentes (como la didáctica) y no solo en instancias curriculares que enseñan a usarlas (como informática), y

b) que dicha utilización sea flexible y permita acompañar la dinámica constante de cambio y aparición/creación de nuevas tecnologías, esto quiere decir, no hacer un uso atado a una herramienta, recurso o software específico o a una modalidad (Cabrera, Cabrera, Carámbula, Pérez y Pérez, 2018).

Esta investigación se asienta en el enfoque clínico y su intención principal consiste en la transformación de una dimensión de la profesión, la práctica, desde sus protagonistas (en especial quienes se preparan para ella). Al decir de Clot y Faïta (2000), se trata de "restablecer el poder de acción de un colectivo profesional" (p. 8). La práctica preprofesional de docentes, convertida en un "objeto de pensamiento" (p. 8), requiere nutrirse de experiencias que guían el mismo; no con recomendaciones sino son actividades que conducen a la reflexión y a la mejora.

\section{Antecedentes}

El uso de tecnologías para mejorar y complementar el análisis y la reflexión durante la formación práctica preprofesional de docentes es una estrategia bastante expandida. Se presenta a continuación una síntesis de aquellas investigaciones que incluyen el uso de videos y/o espacios de aprendizaje asincrónicos.

Rodríguez et al. (2012) realizaron grabaciones de clases de educación primaria en España en el marco de proyectos innovadores de enseñanza de las ciencias experimentales. Luego, las imágenes fueron editadas para seguir un guión. Algunas clases fueron seleccionadas para ser utilizadas libremente, sin guión. De las mismas se identificaron varios aspectos como factibles de análisis: ambiente de la clase, relación con otras asignaturas, trabajo grupal y uso de recursos, entre otros. La observación y análisis de los materiales audiovisuales se realizó de forma conjunta entre profesores y estudiantes en formación para la docencia de las Universidades de Sevilla, Complutense de Madrid y de Cádiz. Destacan las complejidades técnicas que se presentan en la elaboración de un material que sea pedagógicamente potente, y reconocen la importancia de la reflexión ya que la observación y el análisis por sí solos no enriquecen los aprendizajes de la misma manera.

Müller et al. (2013) propusieron el uso de una Videoteca de Buenas Prácticas Docentes como un complemento para mejorar el contacto con prácticas en contextos reales para estudiantes de Pedagogía de la Pontificia Universidad Católica de Chile. Generaron una videoteca que muestra prácticas ejemplares de docentes destacados, acompañadas de guías de trabajo. Dicha videoteca se encuentra disponible para los formadores. Los focos de las guías fueron: el contenido y su didáctica, las interacciones en el aula y el pensamiento de los estudiantes. Los resultados mostraron avances de los estudiantes en la precisión de sus observaciones e interpretaciones así como modificación en la naturaleza y foco de las descripciones. Al igual que Rodríguez et al. (2012) reconocen que, para producir aprendizajes significativos, el uso de videos debe estar mediado por una guía de observación, un docente y un plan de formación, alternado con observación de clases en modalidad presencial.

Hermida (2013) presenta una investigación-acción realizada en el Instituto Cervantes de Hamburgo que conceptualiza la observación de clases como parte de la formación, donde la atención se focaliza en aspectos predeterminados. Analiza los aportes de la auto- 
observación de clases grabadas a la práctica reflexiva y al desarrollo de la autonomía. Concluye que los estudiantes han mejorado la toma de conciencia de sus competencias personales, actitudes y creencias, son capaces de observarse desde una actitud más objetiva y establecen metas de desempeño más apropiadas a su estadío profesional. Karsenti, Collin y Lirai (2012) analizaron el uso de foros y grupos de discusión electrónicos sobre 800 estudiantes durante la práctica preprofesional de formación de docentes en Canadá con el objetivo de mejorar la colaboración entre estudiantes y docentes. Motivó dicha investigación el hecho de que los estudiantes viven diseminados por una vasta área geográfica y que los contextos y tiempos presenciales de la práctica no siempre priorizan o propenden a los intercambios, la reflexión y la colaboración. Identifican aspectos favorables en las propuestas asincrónicas de aprendizaje tales como la accesibilidad y la atención a la diversidad en las formas y tiempos de aprendizaje de cada estudiante. Encuentran que los estudiantes valoran la flexibilidad temporal que otorgan estos recursos para la participación. Concluyen que el uso de estos espacios virtuales de aprendizaje favorece el desarrollo de una cultura de colaboración y minimiza el aislamiento de los estudiantes.

Por su parte, Candela y Viafara (2014) centraron su interés en el conocimiento pedagógico del contenido de estudiantes en formación docente en química en la Universidad de Cali, Colombia, y propusieron el uso de videos de clases para promover la reflexión. Las clases seleccionadas son ejemplares, llevadas a cabo por profesores experimentados. Los autores reconocen que la observación del video por sí sola no es suficiente sino que se hace necesaria la intervención de un formador que conduzca los procesos de reflexión. Proponen alternar el video con el uso de otros materiales y así diversificar las modalidades de trabajo: grupal, subgrupal e individual.

A nivel de la formación profesional de docentes en servicio, Arcavi (2016) del Instituto Weizmann de Ciencias de Israel propuso el desarrollo y amplificación de las destrezas reflexivas de profesores de matemáticas mediante el análisis didáctico y reflexión de clases filmadas. Ha construido un sitio Web donde se dispone de un banco de clases filmadas y se generaron guías de trabajo que permitirían mayor autonomía en la utilización de aquellas. Identifica como uno de los principales desafíos el que los docentes logren modificar su actitud de aprobación/desaprobación de lo que sucede en la clase hacia una actitud de comprensión y de reflexión productivas.

Sáez y Cortés (2019) desarrollan una investigación sobre los niveles de reflexión que se alcanzan en la formación inicial de profesores de Biología y Geología en Zaragoza, España, en base a una actividad de campo filmada desarrollada en tres modalidades didácticas diferentes. Utilizan como generadores de reflexión la visualización del vídeo y su posterior análisis a través de propuestas escritas y de discusiones grupales. Los investigadores destacan que se alcanzan niveles medio-altos en un escaso porcentaje de los estudiantes involucrados. Ello fue determinado por el grado de vinculación que establecen en sus reflexiones al analizar las filmaciones, entre distintos componentes del conocimiento didáctico del contenido.

Con relación a los antecedentes presentados -así como otros que no se incluyen por falta de espacio-, la presente investigación se distingue por los siguientes elementos: la utilización de filmación de clases no modélicas, la participación de profesores de diversos grados de antigüedad y experiencia, el uso de tecnología de grabación en $360^{\circ}$ y la inclusión de instancias de análisis interdisciplinar. 


\section{Metodología}

El equipo de investigación está conformado por tres docentes expertas con más de 20 años de experiencia en formación de profesores de educación media.

El paradigma sobre el que se funda esta investigación es cualitativo y su finalidad es crítica: busca identificar un potencial de cambio (Sandín, 2003). Persigue la interpretación por medio del estudio de unos pocos casos generados de forma cuasi-experimental, con participantes intencionalmente seleccionados. La instancia cuasi-experimental se define como un evento provocado donde no se controlan todas las variables, y que se aplica sobre grupos intactos que ya existían antes del experimento, no se crean para él (Hernández, Fernández y Baptista, 2010). Si bien los cuasi-experimentos se utilizan generalmente en diseños cuantitativos, en esta investigación no se pretende explicar sino valorar cualitativamente un dispositivo de formación. Se introduce una causa experimental (la unidad fílmica y una guía de uso) pero el efecto no es cuantificado sino interpretado. Se alcanza la validación interna sobre la muestra y no se establece causalidad o correlaciones sino comprensión de los fenómenos observados. Los posibles hallazgos tratan de particularidades que aportan comprensión sobre fenómenos educativos y ayudan a iluminar la generalidad (Hernández, Fernández y Baptista, 2010).

El diseño de la investigación consistió en la filmación de clases, la elaboración de unidades fílmicas a partir de ellas y el diseño de protocolos que actuarían de guías del análisis didáctico y reflexión. Por último, se llevaron a cabo las instancias cuasi- experimentales con formadores y estudiantes de formación docente para educación secundaria.

La filmación en $360^{\circ}$ de las clases en educación secundaria se llevó a cabo con profesores voluntarios de las instituciones autorizadas. Las unidades fílmicas son segmentos de las clases, que se elaboraron de acuerdo a los focos de los protocolos de análisis didáctico y reflexión previamente seleccionados. Estos protocolos podían o no ser utilizados por los formadores para guiar las actividades con la unidad fílmica seleccionada. El diseño giró en torno a focos identificados por otros investigadores: macro y micro decisiones (Edelstein, 2011), dimensiones de la clase (Arcavi, 2016), trabajo en grupos (De Zavaleta, 2013), microclases (Anijovich, 2009), incidentes críticos (Anijovich y Cappelletti, 2018), entre otros. Contienen una introducción teórica, las modalidades previstas para el trabajo con la unidad fílmica, la guía de reflexión y las referencias bibliográficas.

Las clases de enseñanza media no debían ser preparadas especialmente para la filmación. Se cumplieron todos los requisitos legales del país para la fijación y uso de imagen de personas mayores y menores de edad. De un total de 15 profesores voluntarios, los 10 primeros manifestaron su interés al tomar conocimiento de la iniciativa. Al progresar la investigación se requirió la selección más afinada de los últimos cinco para lograr contemplar diversidad en cuanto a tipo de institución de educación media (liceo diurno o nocturno, escuela técnica, escuela agraria), ubicación geográfica (en la capital departamental, fuera de ella o en el área rural), nivel (medio básico o medio superior) y área del conocimiento (especialidades científicas, humanidades, matemáticas, artísticas, técnicas).La selección de la unidad fílmica y del protocolo a ser utilizados en la observación, análisis y reflexión en el marco de las instancias cuasi-experimentales fue realizada por el equipo de investigación en diálogo con los formadores. Se les aclaró que podían optar por no utilizar el protocolo y seguir su propia planificación del trabajo con la unidad fílmica. Un total de seis grupos de estudiantes de profesorado $(n=61)$, orientados por formadores de didáctica $(n=8)$ realizaron análisis didáctico y reflexión sobre diferentes unidades fílmicas (una unidad fílmica cada grupo), utilizando opcionalmente los protocolos que guiaban el trabajo con estas. 
La muestra de formadores fue intencional. La selección fue en el primer caso por informante calificado (1) y luego se dio por el fenómeno bola de nieve. Los siete restantes se seleccionaron dentro de los voluntarios por su experticia. Se contempló a la vez la diversificación en las áreas del conocimiento y las instituciones donde ejercían. Por estar sometidos a una instancia cuasi-experimental se les solicitó la firma de un consentimiento informado de participación.

Para la recolección de datos se realizaron observaciones no participantes de las instancias cuasi-experimentales, análisis de contenido de foros y documentos, encuestas en línea y entrevistas en profundidad a docentes y estudiantes. Cada caso se construyó en torno a una instancia cuasi-experimental. Los datos de cada caso provienen de la observación no participante (cuando fue factible), de las entrevistas a sus docentes y estudiantes y del estudio de los documentos que le corresponden. Las encuestas fueron analizadas transversalmente por ser anónimas.

Para valorar los logros de aprendizaje se sistematizaron datos sobre los niveles de reflexión alcanzados (prerreflexión, superficial, pedagógico y crítico). El instrumento utilizado para ello fue diseñado a partir de Lamas y Vargas-D'Uniam (2016).

Todos los materiales producidos en la investigación tienen una licencia Creative Commons de Reconocimiento, NoComercial, Compartirlgual (CC BY-NC-SA).

\section{Criterios de validez y rigor científico aplicados}

Mientras se diseñaban las unidades fílmicas y los protocolos se realizó su pretesteo con una estudiante del último año de formación de profesorado quien, asimismo, cuenta con experiencia docente. Los protocolos estuvieron basados en categorías tomadas de investigadores que han sido reseñados. Cada unidad fílmica contó con la aprobación del profesor filmado, previo a su utilización.

Para minimizar la posibilidad de implicancia ninguno de los casos se desarrolló con grupos de estudiantes entre cuyos docentes se encontraran las investigadoras del presente trabajo. Ninguno de los docentes de didáctica estaba sujeto a relaciones jerárquicas con las investigadoras.

En los casos en que no se realizó observación esto se debió a que el desarrollo era virtual (casos 2 y 3) o en razón de las grandes distancias con respecto al centro de estudio, lo que hacía dificultosa la concurrencia en la fecha de la instancia. Para sostener la confiabilidad de las conclusiones, arribar con solidez a la saturación y lograr la profundidad necesaria en todos los casos se aseguró el contar con documentos (consignas de los docentes, foros de intercambio entre estudiantes) y siempre se llevó adelante una entrevista o más. El instrumento para valorar los niveles de reflexión fue previamente revisado y validado por la experta asesora de la investigación Rebeca Anijovich. Esta investigadora validó, asimismo, los protocolos de análisis y reflexión didácticos.

La herramienta que guiaba la observación no participante fue testeada por el equipo en la primera instancia cuasi-experimental y no necesitó ajustes. 
Aplicación de tecnología en la práctica de la formación inicial de profesores. Valoración del análisis...

\section{Resultados}

Se filmaron 15 clases, en cuatro instituciones de enseñanza media de dos ciudades del Departamento de San José (Uruguay) y de una zona rural. Se abarcaron niveles de enseñanza media básica, técnica y superior en doce asignaturas diferentes. De las clases filmadas se extrajeron 37 unidades fílmicas de duración variable (de 1 a 45 minutos). Se diseñaron nueve protocolos de trabajo con ellas, con diversos focos: trabajo grupal, incidente crítico (dos protocolos diferentes), uso del pizarrón, toma de decisiones, autoobservación, microclases, trabajo práctico. Los protocolos combinan, asimismo, diferentes modalidades de trabajo: presencial/virtual, individual/grupal, sincrónico/ asincrónico, disciplinar/interdisciplinar.

Se realizó observación no participante en tres instancias cuasi-experimentales, análisis de contenido de foros en dos plataformas y de cuatro documentos producidos por los estudiantes, aplicación de encuestas en línea y entrevistas a docentes y estudiantes.

Las seis instancias cuasi-experimentales se desarrollaron en cuatro instituciones de formación de docentes, tanto para modalidad presencial como semipresencial. Participaron ocho formadores de didáctica, tres en una instancia en la que se trabajó en equipo y el resto en forma individual, de las cuales cuatro fueron con estudiantes de una sola especialidad y dos fueron interdisciplinares. Se contó con estudiantes de todos los grados de profesorado. Las especialidades a las que pertenecían los docentes fueron: biología, inglés, matemática, idioma español y literatura. En la Tabla 1 se indican las técnicas y medios de recolección de datos en cada instancia.

Tabla 1. Técnicas de recogida de datos para cada instancia cuasi-experimental

\begin{tabular}{|l|l|l|l|l|l|l|}
\hline & \multicolumn{6}{|c|}{ CASOS } \\
\hline TÉCNICAS & $\mathbf{1}$ & $\mathbf{2}$ & $\mathbf{3}$ & $\mathbf{4}$ & $\mathbf{5}$ & $\mathbf{6}$ \\
\hline Observación no participante & & & & & & \\
\hline Análisis de contenido & & & & & & \\
\hline Entrevista a docente/s & & & & & & \\
\hline Entrevista a estudiante/s & & & & & & \\
\hline Encuesta & & & & & & \\
\hline
\end{tabular}

En dos de las instancias los docentes de didáctica utilizaron el protocolo facilitado por el equipo de investigación; en una de ellas el docente le introdujo modificaciones; en el resto de las instancias (tres) los docentes de didáctica utilizaron una guía de análisis propia.

Ningún docente propuso condiciones para el tipo, duración o procedencia de la unidad fílmica y solo uno encontró posibles mejoras al contenido pedagógico de la unidad fílmica al manifestar que, de los 15 minutos de la misma, solo había aprovechado tres. Seis de los ocho coinciden en que las mismas son mejorables desde el punto de vista audiovisual. De los seis casos cuasi-experimentales, cuatro combinaron las formas de trabajo presencial/virtual, individual/grupal, sincrónico/asincrónico. Esto se produjo en diferentes momentos de la instancia cuasi-experimental: antes, durante o después del uso de los materiales. 
Tabla 2. Características de las instancias cuasi-experimentales (casos)

\begin{tabular}{|c|c|c|c|c|c|c|}
\hline Caso & $\begin{array}{c}\text { Modalidad } \\
\text { de la } \\
\text { carrera }\end{array}$ & $\begin{array}{c}\mathbf{N}^{\circ} \text { de } \\
\text { estudiantes }\end{array}$ & $\begin{array}{c}\text { Estudiantes } \\
\text { de: }\end{array}$ & $\begin{array}{c}\text { Docentes } \\
\text { de: }\end{array}$ & $\begin{array}{c}\text { Unidad } \\
\text { fílmica }\end{array}$ & Protocolo \\
\hline 1 & Presencial & 8 & Inglés & $\begin{array}{c}\text { Didáctica I } \\
\text { de Inglés }\end{array}$ & $\begin{array}{c}\text { Clase de } \\
\text { inglés }\end{array}$ & $\begin{array}{c}\text { Diseño de la } \\
\text { formadora }\end{array}$ \\
\hline 2 & $\begin{array}{c}\text { Semi- } \\
\text { presencial }\end{array}$ & 4 & Matemática & $\begin{array}{c}\text { Didáctica } \\
\text { I de } \\
\text { Matemática }\end{array}$ & $\begin{array}{c}\text { Clase de } \\
\text { matemática }\end{array}$ & $\begin{array}{c}\text { Clase } \\
\text { completa, } \\
\text { adaptado por } \\
\text { la formadora }\end{array}$ \\
\hline 3 & $\begin{array}{c}\text { Semi- } \\
\text { presencial }\end{array}$ & 8 & Biología & $\begin{array}{c}\text { Didáctica II } \\
\text { de Biología }\end{array}$ & $\begin{array}{c}\text { Clase de } \\
\text { biología }\end{array}$ & $\begin{array}{c}\text { Diseño de la } \\
\text { formadora }\end{array}$ \\
\hline 4 & $\begin{array}{c}\text { Presencial } \\
\text { frester- }\end{array}$ & 15 & $\begin{array}{c}\text { Didáctica III } \\
\text { de Inglés, } \\
\text { Disciplinar } \\
\text { y Biología }\end{array}$ & $\begin{array}{c}\text { Clase de } \\
\text { biología }\end{array}$ & $\begin{array}{c}\text { Diseño de los } \\
\text { formadores }\end{array}$ \\
\hline 6 & $\begin{array}{c}\text { Presencial } \\
\text { presencial }\end{array}$ & 6 & Literatura & $\begin{array}{c}\text { Didáctica } \\
\text { Il de } \\
\text { Literatura }\end{array}$ & $\begin{array}{c}\text { Clase de } \\
\text { literatura }\end{array}$ & Incidente \\
crítico
\end{tabular}

La encuesta se aplicó a los 61 estudiantes y a los 8 formadores, de los cuales 32 y 7 , respectivamente, la completaron. Cuatro de los siete docentes respondientes optan por la respuesta: “Debería incorporarse sistemáticamente como una modalidad más, en cualquier momento del año y de la carrera". Cinco consideran que los materiales generaron condiciones de acceso a la reflexión y cuatro de ellos, que permiten profundizar en los niveles de reflexión. Según seis de los encuestados el trabajo con la unidad fílmica contribuiría al acercamiento a las vivencias de los estudiantes en el marco de formación docente y a los aportes teóricos de la didáctica. En relación a los protocolos diseñados por el equipo para guiar la reflexión y el análisis del material fílmico, los siete afirman que con ellos se conseguiría mejorar los niveles de reflexión. Una formadora expresó: "A través del intercambio grupal y el trabajo colaborativo llegaron a reflexiones más profundas que cuando trabajan individualmente, pero que enriquecen el aprendizaje de cada estudiante. La mirada a otro, en este caso desconocido, les brindó la oportunidad de ser muy objetivos y espontáneos. Me parece una estrategia o aporte valioso para incorporarlo a las clases para el desarrollo de la reflexión crítica de los estudiantes de formación docente" (respuesta de pregunta abierta en la encuesta).

En las respuestas a la encuesta por parte de los estudiantes destacan como fortalezas de las unidades fílmicas y de las guías de trabajo el generar condiciones de acceso a la reflexión $(n=26)$ y permitir identificar adecuadamente los sucesos que ocurren en el aula $(\mathrm{n}=19)$. Como debilidades destacan los aspectos técnicos de las unidades fílmicas $(n=20)$ y la posibilidad de que la cámara altere el normal desarrollo de una clase $(n=20)$. Indican que el trabajo con la unidad fílmica los acercó a: las emociones y percepciones sobre la profesión que han elegido $(n=19)$ y a los aportes teóricos de la didáctica $(n=18)$. La mayoría considera que, en sus condiciones actuales, alternar períodos de análisis de clases filmadas con práctica presencial implicaría una mayor flexibilidad horaria para realizar algunas de las tareas de aprendizaje al cursar Didáctica I y II $(n=20)$. Dieciocho 
estudiantes también opinan que otorgan la posibilidad de sortear posibles obstáculos tales como la momentánea falta de formadores (profesor adscriptor, profesor de didáctica), imposibilidad de viajar (enfermedad, razones familiares debidamente justificadas, falta de transporte), entre otros. Asimismo, 17 seleccionan la posibilidad de apoyo de otros compañeros o la posibilidad de ayudarse entre sí al realizar trabajos grupales.

En las entrevistas, formadores y estudiantes afirman que no es un recurso para utilizar en forma exclusiva sino en combinación con otras modalidades. Encuentran que el trabajo con las unidades fílmicas propicia la colaboración al permitir el trabajo grupal y hace de la práctica preprofesional una actividad más accesible, diversa e inclusiva. Sin embargo, no aparecen menciones al uso pedagógico de las tecnologías o a la búsqueda de nuevos horizontes que trascienden lo didáctico hacia lo crítico: la accesibilidad, la atención a la diversidad, cuestiones de género, barreras de aprendizaje, infraestructura edilicia y acondicionamiento del salón (luz, acústica, temperatura, distribución de bancas), entre otros.

De acuerdo a las producciones de los estudiantes (documentos y foros) compartidas por los formadores con el equipo de investigación, y a la observación no participante de las instancias cuasi-experimentales, los estudiantes alcanzarían niveles de reflexión variables por lo que se cree que las unidades fílmicas y las modalidades de trabajo que se propusieron propician el aprendizaje y se adecuan a la capacidad de análisis en diferentes grados de avance en la carrera. "Me siento más consciente de lo que hago en mis clases" manifestó un estudiante (E1OBS).

Si bien aún no se ha culminado el estudio de todos los materiales, en la Tabla 3 se presenta un avance de tres de los casos. 
Tabla 3. Niveles de reflexión alcanzados por los estudiantes

\begin{tabular}{|c|c|c|c|}
\hline Caso & Nivel* & Fuente & Evidencia \\
\hline 5 & Prerreflexivo & $\begin{array}{l}\text { Material sintético grupal } \\
\text { entregado por los } \\
\text { estudiantes al finalizar la } \\
\text { actividad con la unidad } \\
\text { fílmica. }\end{array}$ & $\begin{array}{l}\text { "No se ha establecido un cierre } \\
\text { evidentemente el conocimiento que se } \\
\text { venía construyendo ha quedado inconclusc } \\
\text { o por lo menos suspendido durante algúr } \\
\text { tiempo. (...) Con el corte abrupto no se } \\
\text { cierra el tema y tal vez se pierda el eje o } \\
\text { hilo estructurador. Esto puede causar una } \\
\text { confusión en el estudiante o la necesidac } \\
\text { de reiterar buena parte de lo ya hecho, para } \\
\text { poder continuar el proceso de la adquisiciór } \\
\text { de ese conocimiento en la clase o en la hora } \\
\text { siguiente". }\end{array}$ \\
\hline 2 & Superficial & $\begin{array}{l}\text { Trabajo entregado por } \\
\text { una estudiante al finalizar } \\
\text { la actividad con la unidad } \\
\text { fílmica. }\end{array}$ & $\begin{array}{l}\text { "De las observaciones de la videoclase en } \\
\text { cuestión se registran interacciones, diálogos } \\
\text { entre la docente y los alumnos en forma } \\
\text { continua. Los alumnos trabajan en grupos } \\
\text { de dos o tres en una actividad de función } \\
\text { de segundo grado. La profesora luego de } \\
\text { entregarles el impreso con la actividad los } \\
\text { deja que trabajen solos, casi sin indicaciones } \\
\text { salvo las que están escritas en la actividad" }\end{array}$ \\
\hline 1 & Pedagógico & $\begin{array}{l}\text { Observación no } \\
\text { participante (apuntes de } \\
\text { la investigadora) }\end{array}$ & $\begin{array}{l}\text { "Los estudiantes plantean preguntas } \\
\text { cómo decide el profesor si una estrategia } \\
\text { está siendo efectiva o no, y cuándo decide } \\
\text { cambiarla. El análisis del uso del tiempo } \\
\text { se basa en cómo la docente lo administra } \\
\text { para asegurar la mayor cantidad posible } \\
\text { de interacciones con y entre los alumnos". }\end{array}$ \\
\hline
\end{tabular}

* Como afirman Lamas y Vargas-D'Uniam (2016), las personas pueden encontrarse en diferentes niveles de reflexión en forma paralela. Lo que aquí se expone son ejemplos ilustrativos dentro de los casos.

En la Tabla 3, en relación al caso 5, se ha categorizado el nivel de prerreflexión dado que los estudiantes sostienen una visión lineal y simple de los procesos de enseñanza. En el caso 2, corresponde a un nivel superficial debido a que la estudiante realiza una descripción basada en las estrategias didácticas utilizadas por el docente filmado para alcanzar sus objetivos de enseñanza. En el caso 1, se le adjudica el nivel pedagógico dado que los estudiantes establecen relaciones con conocimientos didácticos teóricos y han reflexionado en torno a la importancia de las decisiones del docente sobre los resultados de aprendizaje de sus alumnos.

Se ha creado un sitio Web con un repositorio abierto con las unidades fílmicas (http:// recyreap.cfe.edu.uy) y se ha dispuesto, junto a ellas, la variedad de protocolos diseñados por el equipo. Cualquier docente interesado, así como los lectores de este artículo, pueden acceder al sitio Web del proyecto para observar las unidades fílmicas en $360^{\circ}$ y los protocolos de reflexión. 


\section{Discusión}

Los resultados indicarían que los docentes valoran favorablemente los dispositivos probados como un complemento a la modalidad de formación actual. Los docentes valoraron más las unidades fílmicas que los instrumentos de reflexión y análisis propuestos. De hecho, solo dos de las seis instancias cuasi-experimentales se llevaron a cabo con protocolos propuestos por el equipo de investigación. Es posible que no sea una cuestión de calidad sino de las competencias necesarias para producir el material audiovisual y las tareas suplementarias y diversas que implican (autorizaciones institucionales, permisos de uso de imagen, coordinación con los voluntarios). Por tanto, si son recursos tan valiosos debería mejorarse la calidad de vídeo y de audio de las unidades fílmicas, colocar la cámara en lugares estratégicos, estudiar con anticipación la disposición del escenario (el aula, el laboratorio) con el fin de lograr la mejor toma de imágenes y sonidos y disminuir las interferencias. Esta dificultad ya ha sido identificada por otros investigadores (Rodríguez et al., 2012).

Los materiales han mostrado adaptarse a diferentes grados de avance en la carrera y a la diversidad de formas de aprender. Podrían utilizarse en diferentes niveles (introductorios, avanzados), constituyéndose así en un recurso flexible y de alta potencialidad que se adecua a las características del grupo y al nivel de profundidad de cualquier currícula. Coincide con las características exitosas identificadas por Vaillant (2019) y con la concepción de inclusión digital genuina de Cabrera et al. (2018). Las unidades fílmicas se adaptarían al público, a sus características, sus expectativas, al foco elegido y a la profundidad que se desee alcanzar. Dichas unidades son un elemento interesante para ser utilizado también en la formación en servicio, con docentes egresados o idóneos (personas que no tienen título docente pero ejercen la docencia a nivel de educación media). Existen antecedentes de dicho uso (Anijovich y Cappelletti, 2018; Arcavi, 2016) aunque no con tecnología $360^{\circ}$, lo que le otorga un punto más a favor de la complejidad de los resultados de aprendizajes obtenidos. Queda como deuda (debido al poco tiempo que lleva en ejecución) un análisis de la sustentabilidad y la evaluación externa de la propuesta, elementos también fundamentales en el éxito identificados por Umpiérrez y Rodríguez (2017).

Tanto los formadores como los estudiantes consideran que, a través de las respuestas a la encuesta, el trabajo con la unidad fílmica los acerca a la dimensión teórica de la didáctica. Esto contribuiría al necesario y deseable diálogo teoría-práctica que se reclama en la formación de docentes. Ambos tipos de conocimiento son necesarios para las prácticas: el inmediato, utilizado cotidianamente, y las construcciones teóricas formales. Desde esta perspectiva, el conocimiento académico "constituye un instrumento de reflexión cuando se integra no como información fragmentaria sino como parte de los esquemas de pensamiento que activa una persona al interpretar la realidad concreta en la que vive y sobre la que actúa..." (Edelstein, 2011, 108). La dimensión reflexiva constituye un medio para reconstruir críticamente la teoría, en la práctica.

Docentes y estudiantes coinciden en la necesidad de la presencia de un formador acompañando y guiando la reflexión, de la alternancia con observación presencial y de materiales orientadores. Asimismo, los estudiantes reconocen mayormente que con los dispositivos experimentados se lograría mayor flexibilidad horaria en la formación práctica de la carrera. Esto coincide con los hallazgos de otros investigadores (Müller et al., 2013; Rodríguez, et al. 2012; Karsenti, Collin y Lirai, 2012). 
Hasta el momento no se han hallado evidencias de estudiantes que hubieran realizado reflexiones de nivel crítico, tal como sucedió en el estudio de Lamas y Vargas-D'Uniam (2016). Esta afirmación es preliminar ya que no se ha finalizado el análisis de todos los materiales de los seis casos. Sin embargo, hasta ahora no han surgido reflexiones políticas, sociales, éticas o emocionales relacionadas con las actividades de enseñanza desarrolladas. Existirían varias causas posibles: una agenda didáctica que soslaya estas temáticas; el perfil de los docentes que participaban; el contenido de las unidades fílmicas o de los protocolos. Karsenti, Collin y Lirai (2012), que estudiaron la naturaleza de los intercambios de estudiantes en formación inicial docente a través de medios virtuales, hallaron que puede constituirse en un lugar para compartir las experiencias personales y profesionales, darse aliento e intercambiar materiales. Por tanto, se debe realizar un esfuerzo para que a las bondades del trabajo a distancia se sumen la reflexión crítica, la articulación teórica y la complejidad del análisis.

\section{Conclusiones}

Esta investigación persigue el objetivo general de enriquecer y diversificar la formación práctica de docentes. Se han valorado las potencialidades formativas de utilizar clases filmadas en $360^{\circ}$ en centros de enseñanza media, en el análisis didáctico de dicha formación. Esto se llevó adelante mediante la sistematización de las percepciones de ese uso en docentes y estudiantes participantes en instancias cuasi-experimentales y del relevamiento de los niveles de reflexividad alcanzados en el análisis didáctico durante las mismas.

Las percepciones recogidas indican que el trabajo mediado por clases filmadas, no modélicas, de profesores con los cuales no tienen vínculo ni afectivo ni profesional establece un ambiente de bajo riesgo, que estimula la libertad de expresión, los intercambios y cuestionamientos que conducen a más aprendizajes. Esta modalidad permite cambiar el ángulo de observación, focalizar y profundizar, volver sobre un mismo evento y reinterpretarlo a la luz de esas diferentes perspectivas. El trabajo de análisis sobre clases filmadas puede constituirse en un valor agregado a la formación, otorga nuevas modalidades de trabajo y proporciona oportunidades de aprendizaje que no están presentes en la observación de clases presenciales.

Las clases filmadas resultan en infinidad de unidades fílmicas que se adecuan al nivel de análisis que el formador encuentre conveniente. Las mismas son de diferente duración y muestran diferentes situaciones pasibles de análisis. Cada unidad puede observarse desde diferentes ángulos, por lo que se puede analizar qué hace el profesor y qué hacen los alumnos al mismo tiempo. Una unidad fílmica puede ser seleccionada para analizar el uso del pizarrón pero esa misma puede ser utilizada proponiendo a los practicantes que extraigan de ella un incidente crítico. Por tanto, la filmación de una clase en enseñanza media proporciona innumerables oportunidades de abordaje, de nuevos análisis y aprendizajes.

En cuanto a los niveles de reflexión alcanzados puede afirmarse que, para los casos estudiados, pudo generarse reflexión durante el análisis didáctico mediado por las filmaciones en $360^{\circ}$, por lo que se estaría ante un recurso de enseñanza innovador, valioso, que requiere ser más estudiado y expandido para reafirmar sus potencialidades. El hecho de que no se haya arribado al nivel de reflexión crítica puede deberse a los dispositivos en sí mismos. Sería necesario mejorar las unidades fílmicas, lograr la emergencia de temas más comprometidos que están presentes en la agenda didáctica: lo social, lo político, lo ético y lo emocional en clase. Son temas controvertidos sobre los cuales es valioso que 
los docentes en formación reflexionen. Surgen entonces nuevos desafíos, que el equipo se está planteando actualmente y que consisten en filmar clases en ambientes diversos o menos accesibles con el fin de enriquecer el repositorio: formación en servicio, educación inicial de cero a tres años, educación en contextos de privación de libertad, escuelas especiales que acogen a personas con discapacidad, aulas hospitalarias, educación de adultos, entre otras.

Este proyecto es más pragmático que teórico por lo que los esfuerzos por potenciar el repositorio consisten en posicionarlo como herramienta útil para el aprendizaje más que en explicitar formas específicas de uso. Si bien el equipo se vuelca hacia el análisis didáctico desde una postura reflexiva, no se pretende adherir a una forma determinada de uso de estos recursos, protocolos y unidades fílmicas $360^{\circ}$. Se desea abrir el campo de la innovación y habilitar usos no tradicionales, experimentales, que sirvan para incorporar dimensiones interesantes que interpelan actualmente a todos los formadores. Por esta razón se aspira a difundir los recursos como accesibles, diversos, integradores, económicos y que aportan a mejorar las trayectorias. La potencia de estos recursos y su alcance está en manos de los docentes y los estudiantes que deseen utilizarlos, adecuándolos a situaciones, inclinaciones, epistemologías, preferencias y contextos de aprendizaje que el equipo de investigación no puede prever a priori. La diversidad de aproximaciones teóricas posibles hizo que se priorizara la disponibilidad de las unidades fílmicas más que la forma y modalidades de uso. Los protocolos propuestos en este proyecto tienen el objetivo de valorar el potencial de las unidades fílmicas como recurso de aprendizaje, no de marcar cómo deben utilizarse.

Emergen nuevas preguntas que podrían abrir el camino a otras investigaciones: ¿Cuáles son las condiciones óptimas de obtención de buenas unidades fílmicas, desde el punto de vista técnico? ¿y desde el punto de vista pedagógico? ¿Qué otras aplicaciones y herramientas tecnológicas pueden contribuir a enriquecer y hacer más accesible la práctica preprofesional? ¿Cómo pueden aplicarse estas actividades a la reflexión y al análisis de formadores de educación en primera infancia, educación primaria, terciaria y/o universitaria?

Esta investigación contó con el apoyo y asesoramiento en temas técnicos y legales de la Unidad Académica de Tecnología Educativa del Consejo de Formación en Educación, a quienes las investigadoras expresan su agradecimiento. El equipo de investigación manifiesta también el profundo agradecimiento y reconocimiento a los docentes voluntarios filmados, a los alumnos de enseñanza media, a los padres que autorizaron el uso de su imagen y a las direcciones de los centros educativos donde se realizaron las filmaciones. Por último, fue invaluable el apoyo de los docentes de didáctica y de los estudiantes de formación docente que también voluntariamente llevaron adelante las instancias cuasi-experimentales. Vaya para ellos el agradecimiento del equipo. Sin todas estas buenas disposiciones, la investigación no hubiera podido llevarse adelante. 


\section{Referencias bibliográficas}

Anijovich, R. (2009). Transitar la formación pedagógica. Dispositivos y estrategias. Buenos Aires, Argentina: Paidós. http://pdfhumanidades.com/sites/default/files/apuntes/ ANIJOVICH\%20-\%20Transitar\%20la\%20formaci\%C3\%B3n\%20pedag\%C3\%B3gica\%20 -\%20cap\%202.pdf

Anijovich, R. y Cappelletti, G. (2018). Despertar la reflexión en la formación docente: dispositivos efectivos. Innoeduca. International Journal of Technology and Educational Innovation, 4(1), 24-35. DOI: http://dx.doi.org/10.24310/innoeduca.2018.v4i1.3610

Arcavi, A. (2016). El rol del video en el desarrollo profesional del docente de matemáticas en la escuela secundaria. Actas del 6. Congreso Uruguayo de Educación Matemática. Departamento de Enseñanza de las Ciencias. Instituto Weizmann de Ciencias - Rehovot, Israel. https://semur.edu.uy/curem6/actas/pdf/44.pdf

Cabrera, C., Cabrera, A., Carámbula, S., Pérez, A. y Pérez, M. (2018). Tecnologías digitales: análisis de planes de profesorado de Uruguay. Cuadernos de Investigación Educativa, 9 (2). https://revistas.ort.edu.uy/cuadernos-de-investigacion-educativa/article/view/2858/2863

Candela-Rodríguez, B.F. y Viafara-Ortiz, R. (2014). Articulando la CoRe y los PaP-eR al programa educativo por orientación reflexiva: una propuesta de formación para el profesorado de química. Tecné, Episteme y Didaxis: TED. Núm 35. p. 89-111. DOI: https:// doi.org/10.17227/01213814.35ted89.111

Clot, Y. y Faïta, D. (2000). Genres et styles en analyse du travail. Concepts et méthodes. Travailler, 2000 (4), 7-42. http://psychanalyse.cnam.fr/medias/fichier/ texteclot4_1306851012723.pdf

Davini, C. (2015). La formación en la práctica docente. Buenos Aires: Paidós.

De Zavaleta, M. (2013). La importancia del trabajo grupal en el aula. Escritos en la Facultad, 84, 80-82. https://fido.palermo.edu/servicios_dyc/publicacionesdc/vista/detalle_articulo. php?id_articulo $=9174 \&$ id libro $=443$

Edelstein, G. (2011). Formar y formarse en la enseñanza. Buenos Aires: Paidós.

Fernández, J.P. (2016). La adquisición y desarrollo de la competencia digital en alumnos de educación secundaria. Estudio de caso. Cuadernos de Investigación Educativa, 7 (2). 83 98 DOI: https://doi.org/10.18861/cied.2016.7.2.2612

Fernández, T. y Fernández J. (1994). Técnica de trabajo con profesores sobre su práctica docente: «Terapia de Knoll». Investigación en la escuela, 22, 91-103. https:// revistascientificas.us.es/index.php/IE/article/view/8507/7585

Fernández, J., Elórtegui, N. y Medina, M. (2003). Los incidentes críticos en la Formación y Perfeccionamiento del Profesorado de Secundaria de Ciencias de la Naturaleza. Revista Interuniversitaria de Formación del Profesorado, 17 (1), 101-112. https://www.redalyc.org/ pdf/274/27417107.pdf 
Aplicación de tecnología en la práctica de la formación inicial de profesores. Valoración del análisis...

Hermida, A. (2013). Las grabaciones de clase como instrumento para facilitar la reflexión y la autonomía docente. Revista Nebrija de Lingüística Aplicada (2013) 13. https://www. nebrija.com/revista-linguistica/files/articulosPDF/articulo_5326d3db520bb.pdf

Hernández, R., Fernández, C. y Baptista, P. (2010). Metodología de la investigación. México: Mc Graw-Hill. https://www.esup.edu.pe/descargas/dep investigacion/ Metodologia\%20de\%20la\%20investigaci\%C3\%B3n\%205ta\%20Edici\%C3\%B3n.pdf

Karsenti, T., Collin, S. y Lirai, M. (2012). Impacto del uso de los fórums y los grupos de discusión electrónicos durante las prácticas preprofesionales de los estudiantes de educación. Apertura. Revista de innovación educativa, 4(2). http://www.udgvirtual.udg.mx/ apertura//index.php/apertura/article/view/333/294

Lamas, P. y Vargas-D'Uniam, J. (2016). Los niveles de reflexión en los portafolios de la Práctica Pre-Profesional Docente. REDU. Revista de Docencia Universitaria, 14(2), 57-78. https://doi.org/10.4995/redu.2016.5680, https://polipapers.upv.es/index.php/REDU/ article/view/5680/7142

Müller, M., Calcagni, E., Grau, V., Preiss, D. \& Volante, P. (2013). Developing observation skills in pre-service teachers: Results of a pilot intervention based on the use of the Video Library of Good Teaching Practices. Estudios pedagógicos (Valdivia), 39 (Especial), 85-101. https://dx.doi.org/10.4067/S0718-07052013000300007,

https://scielo.conicyt.cl/scielo.php?script=sci_arttext\&pid=S071807052013000300007\&ln g=en\&nrm=iso\&tlng=en

Sandín, M.P. (2003). Investigación cualitativa en educación. Fundamentos y tradiciones. Madrid: Mc Graw-Hill.

Pereyra, A., Moscato, P., Calderón, L. y Oviedo, M. (2016). Análisis de las prácticas docentes desde la didáctica profesional. Buenos Aires: UNIPE, Editorial Universitaria. https://www. researchgate.net/publication/297409258_Analisis_de_las_practicas_docentes_desde_la didactica_profesional

Perrenoud, P. (2007). Desarrollar la práctica reflexiva en el oficio de enseñar. Profesionalización y razón pedagógica. Colección Crítica y fundamentos. Barcelona: Graó. https://coleccion. siaeducacion.org/sites/default/files/files/6_perrenoud_philippe_2007desarrollar_la practica_reflexiva.pdf

Rodríguez, F., Ezquerra, A., Rivero, A., Porlán, R., Azcárate, P., Martín del Pozo, R. y Solís, E. (2012). El uso didáctico del vídeo para aprender a enseñar ciencias. Actas del XXV Encuentro de Didáctica de las Ciencias Experimentales, Santiago de Compostela, 741-746. http://hdl. handle.net/11441/25487

Sáez Bondía, M. J. y Cortés García, A. L. (2019). ¿Cómo evaluar la reflexión sobre la práctica docente? Un ejemplo en la formación inicial del profesorado de Biología y Geología. Didáctica de las ciencias experimentales y sociales, 37, 127-146. DOI: 10.7203/DCES.37.14797 https://ojs.uv.es/index.php/dces/article/view/14797/pdf 
Umpiérrez Oroño, S. y Rodríguez Zidan, E. (2017). Aportes para el diseño de una herramienta para el seguimiento y evaluación de experiencias innovadoras. REXE. Revista de Estudios y Experiencias en Educación, 16 (31), 107-126. http://www.redalyc. org/articulo.oa?id=243152008007

Vaillant, D. (2019). Formación del profesorado para la innovación. En: Martínez, M. y Jolonch, A. (Coords). Las paradojas de la innovación educativa, cap. 11. Barcelona: Horsori. http://www.denisevaillant.com/wp-content/uploads/2019/10/las-paradojas-de-lainnovacion-educativa-denise-vaillant.pdf

\section{(cc) BY}

Este artículo está sujeto a una licencia internacional Creative Commons Attribution 4.0.

${ }^{*, * *}{ }^{* * *}$ IFD de San José, Consejo de Formación en Educación, Uruguay

1'Doctora en Educación y Master en Educación, Universidad ORT Uruguay. Licenciada en Ciencias Biológicas, Universidad de la República (Uruguay). Directora, Instituto de Formación Docente de San José, Administración Nacional de Educación Pública (ANEP). Tutora de postgrados del Instituto de Educación, Universidad ORT Uruguay.

${ }^{2}$ Doctora (Cand.) en Educación, Universidad Internacional Iberoamericana (México).

Magíster en Enseñanza de las Ciencias y Profesora de Biología, CLAEH (Uruguay). Magíster en Educación, Sociedad y Política, Facultad Latinoamericana de Ciencias Sociales (Uruguay). Docente, Instituto de Formación Docente de San José, Consejo de Formación en Educación y Consejo de Educación Secundaria (Uruguay). Formadora en Ciencias Naturales, Instituto de Formación en Servicio del Consejo de Educación Inicial y Primaria.

${ }^{3}$ Analista Programadora, Universidad de la República (Uruguay). Tester de software, Centro de Ensayos de Software (Uruguay). Docente Orientadora en Tecnologías y de Informática, Instituto de Formación Docente de San José (Uruguay).

\section{Notas}

Aprobación final del artículo, editora responsable Mag. Verónica Zorrilla de San Martín Contribución de autoría: Las tres coautoras participaron de forma equitativa en las etapas de diseño de la investigación, recolección de datos, procesamiento, análisis y elaboración del texto.

'Este trabajo recibió apoyo económico de la Agencia Nacional de Investigación e Innovación, la Fundación Ceibal y el Consejo de Formación en Educación de la Administración Nacional de Educación Pública de Uruguay. 